\title{
Magnetic properties and giant magnetoimpedance of surface modified Co-based amorphous ribbons with carbon covering
}

\author{
Elizaveta V. Golubeva ${ }^{1}$, Anna A. Chlenova ${ }^{1}$, Elena A. Stepanova ${ }^{1}$ and Galina V. Kurlyandskaya ${ }^{1,2}$ \\ ${ }^{1}$ UrFU Ural Federal University, Laboratory of Magnetic sensoric, 620002, Lenin Ave. 51, Ekaterinburg, Russia \\ ${ }^{2}$ Universidad del País Vasco UPV-EHU, Depto Electricidad y Electrónica, 48940, Leioa, Spain
}

\begin{abstract}
Fe}_{3} \mathrm{Co}_{60} \mathrm{Cr}_{3} \mathrm{Si}_{10} \mathrm{~B}_{12}$ and $\mathrm{Fe}_{6} \mathrm{Co}_{60} \mathrm{Ni}_{10} \mathrm{Si}_{14} \mathrm{~B}_{10}$ amorphous ribbons were surface modified in toluene at room temperature. Such a treatment resulted in deposition of thin carbon-based layer. As a result of the carbon covering deposition the stress distribution in the near-surface layers was changed due to partial compensation of the initial quenching stresses. Comparative analysis of magnetic and magnetoimpedance properties of as-quenched and surface modified ribbons confirms changes in effective magnetic anisotropy as a result of surface treatment. An increase of the corrosion resistance of the ribbons with carbon covering can be useful for the development amorphous ribbon based magnetic biosensor.
\end{abstract}

\section{Introduction}

Miniature compact analytical devices (biosensors) have been developed for a large range of applications [1]. Biosensors for detection of magnetic markers acting as biomolecular labels are promising for medical diagnostics, drugs delivery, tissue engineering and environmental control [2]. Various working principles for the magnetic particles detection can be mentioned: inductive coils, giant and anisotropic magnetoresistance, Hall effect, giant magnetoimpedance (GMI) [3, 4]. GMI effect consists in a large change of the impedance of a ferromagnetic conductor $(\mathrm{Z})$ under application of the magnetic field [5]. Due to high sensitivity GMI effect is widely used for the development magnetic field sensors [6-7]. High frequency impedance depends on the classic skin penetration depth $(\delta)$. In magnetic materials, the application of a magnetic field changes the magnetic permeability, skin depth and the total impedance: $\mathrm{Z}=$ $Z(\delta)$. As the effect depends on the effective magnetic anisotropy, properties of a surface layer become very important. Amorphous ribbons are cheap materials with high GMI sensitivity, some of them having good corrosion resistance [4,8]. They were proposed as disposal sensitive elements for biosensors. However, surface functionalization of the metallic glass is difficult. Stability of the sensitive element in the biocorrosive environments is also important. It can be done by polymer covering [9] but good adhesion between the ribbon and polymer is not always a case.

Defected graphene-like carbon layer can be used to increase adhesion and to protect samples from the corrosion. Room temperature carbon deposition was discovered for the case of $\mathrm{Fe}$ and $\mathrm{Ni}$ nanoparticles and
$\mathrm{Fe}$ and FeNi films [10]. Heterogeneous Scholl oxidation of the aromatic hydrocarbons was proposed as possible mechanism of the reaction with the first step being an oxidation of atoms of metals by oxygen dissolved in the solvent. No data on low temperature carbon deposition in aromatic solvents were reported for the case of amorphous materials. The main objective of this work was to study the possibility and particular features of the process of ambient conditions of carbon deposition onto the surface of amorphous ribbons with GMI responses.

\section{Experimental methods}

Rapidly quenched Co-based amorphous ribbons of two compositions were studied: $\mathrm{S} 1-\mathrm{Fe}_{3} \mathrm{Co}_{66} \mathrm{Cr}_{3} \mathrm{Si}_{16} \mathrm{~B}_{12}(0.8$ $\times 0.025 \mathrm{~mm})$ and $\mathrm{S} 2-\mathrm{Fe}_{6} \mathrm{Co}_{60} \mathrm{Ni}_{10} \mathrm{Si}_{14} \mathrm{~B}_{10}(1.3 \times 0.025$ $\mathrm{mm})$. Surface modification of the samples was done in the toluene at ambient conditions for one or two months period. Toluene was used because the best results in previous experiments were observed with a use of it [10]. Table 1 lists all samples and their states. Scanning electron microscopy (SEM) and energy dispersive x-ray spectroscopy (EDX) were used for structural characterization and elemental analysis (AURIGA CrossBeam equipment). For mass losses determination ME 235S analytical balance was used. Relative weight loss is determined by follows: $\Delta m / m_{0}=\left(m_{0}-m_{i}\right) / m_{0}$. Where $m_{0}$ is a mass of the sample in initial state and $m_{i}$ is a mass of the sample after i-days exposure in $0.9 \% \mathrm{NaCl}$ solution.

Magnetic properties were measured by MMKS 100-05 installation. Visualization of domain structure was done with EVICO magnetooptical Kerr-microscope using the equatorial effect (MOKE). The complex impedance ( $\mathrm{Z}$ ) was measured as a function of the external magnetic field $(\mathrm{H})$ applied in the direction of the flowing

\footnotetext{
Corresponding author: anna.chlenova@urfu.ru
} 
alternating current and parallel to the long side of the ribbon in a frequency range $1-50 \mathrm{MHz}$. Measurements were made in a "microstripe" line by standard methodology [11]. The GMI ratio was defined as follows: $\Delta \mathrm{Z} / \mathrm{Z}=\left[\mathrm{Z}\left(\mathrm{H}_{\mathrm{o}}\right)-\mathrm{Z}\left(\mathrm{H}_{\max }\right)\right] / \mathrm{Z}\left(\mathrm{H}_{\max }\right)$, where $\mathrm{H}_{\max }=$ $100 \mathrm{Oe}$ is maximum applied magnetic field. Maximum values of the total impedance $\left((\Delta \mathrm{Z} / \mathrm{Z})_{\max }\right)$ were also used for analysis.

Table 1. Designations of the samples used in this work.

\begin{tabular}{c|c|c}
\hline Composition & Name & $\begin{array}{c}\text { Time of exposure in } \\
\text { toluene (months) }\end{array}$ \\
\hline \multirow{2}{*}{$\mathrm{Fe}_{3} \mathrm{Co}_{66} \mathrm{Cr}_{3} \mathrm{Si}_{16} \mathrm{~B}_{12}$} & $\mathrm{~S} 1-0$ & 0 \\
& $\mathrm{~S} 1-1$ & 1 \\
& $\mathrm{~S} 1-2$ & 2 \\
\hline & $\mathrm{S} 2-0$ & 0 \\
$\mathrm{Fe}_{6} \mathrm{Co}_{60} \mathrm{Ni}_{10} \mathrm{Si}_{14} \mathrm{~B}_{10}$ & $\mathrm{~S} 2-1$ & 1 \\
& $\mathrm{~S} 2-2$ & 2 \\
\hline
\end{tabular}

\section{Results and discussions}

The structure and elemental composition of the samples were analyzed in as-prepared state and after exposure in toluene for two months. Fig. 1 and Table 2 show selected examples. The morphology of as-prepared ribbons was typical for this kind of materials. It was observed differences in the compositions before and after surface modification: carbon and oxygen content of the surface layer was significantly increased after modification. Moreover, an appearance of dark circle areas on SEM images of the sample after the treatment can be noticed on Fig.1 (b). Similar defects corresponding to areas an accumulation of carbon were also observed in previous experiments with thin films [10]. Fig.1(c) shows the effect of carbon re-distribution as a result of covering interaction with electron beam during SEM studies (black squares are previously scanned areas). Observed compositional changes are consistent with proposed mechanism of the low temperature carbon deposition [10].

The next step is to understand the role of the surface modification in the change of magnetic properties. Figure 2 shows that the general shape of the $\mathrm{M}(\mathrm{H})$ loops was not changed by the surface modification: only a small reduction of the coercivity takes place in the S2 ribbon. In all cases the effective magnetic anisotropy was clearly a longitudinal one with possible small contribution of a transverse anisotropy (slow saturation approach). Fig. 3-4 show changes of magnetic domains under application of the magnetic field. For analysis of the magnetization process we must take into account both measurements of bulk $\mathrm{M}(\mathrm{H})$ loops and MOKE data. In the initial state of sample S1 there is a surface contribution of the transverse magnetic anisotropy (perpendicular to the ribbon axis bar domain structure). After the surface modification transverse and longitudinal components begin to compete in close to surface layer. In the S2 case the elongated bar domains parallel to the ribbon axis confirm that the main contribution in the initial state is the longitudinal magnetic anisotropy. After the treatment domains become invisible due to the higher surface roughness. As those changes took place in a thin $(\approx 20 \mathrm{~nm})$ surface layer, mentioned effects are absent in the $\mathrm{M}(\mathrm{H})$ loops.

Table 2. Percentage elemental composition of S1 and S2 ribbons before and after modification in toluene for 2 months.

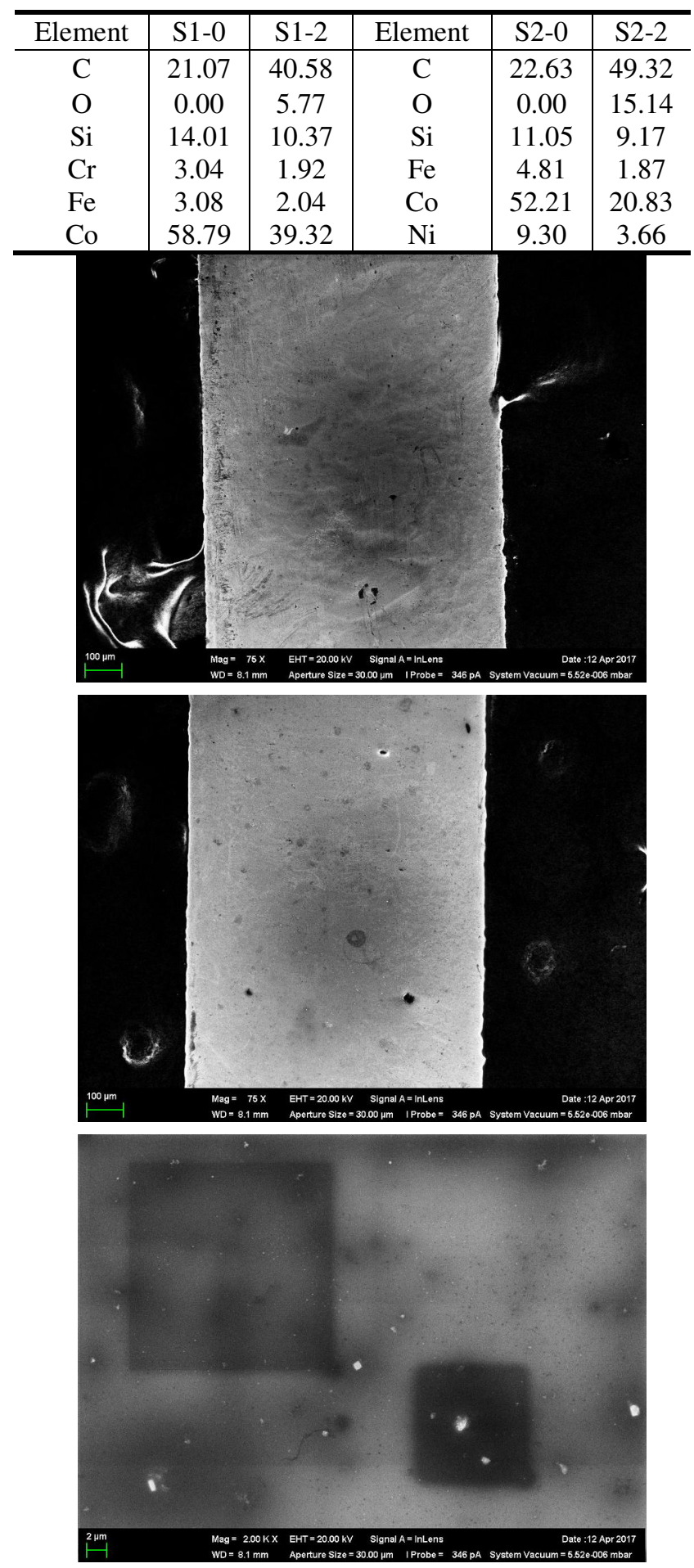

Fig. 1. SEM images of the $\mathrm{FeCoCrSiB}$ ribbon before (a) and after $(b, c)$ surface modification in toluene for 2 months.

Frequency dependencies for $\Delta \mathrm{Z} / \mathrm{Z}_{\max }$ ratio are shown in Figure 5. Decrease of the value of $\Delta Z / Z_{\max }$ and its shift toward the higher frequencies as a result of surface modification were observed. Fig. 6 shows field 
dependences of the GMI ratios. For both samples the one-peak shape of the curves was not changed as a result of the surface modification, but fine changes are evident in the small fields. In the case of S1 ribbon both decrease of a distance between GMI peaks and «down» (decreasing) and «up» (increasing field) branches take place. In the S2 case (Fig. 6(c)) there is also a decrease of a distance between «down» and «up» branches.
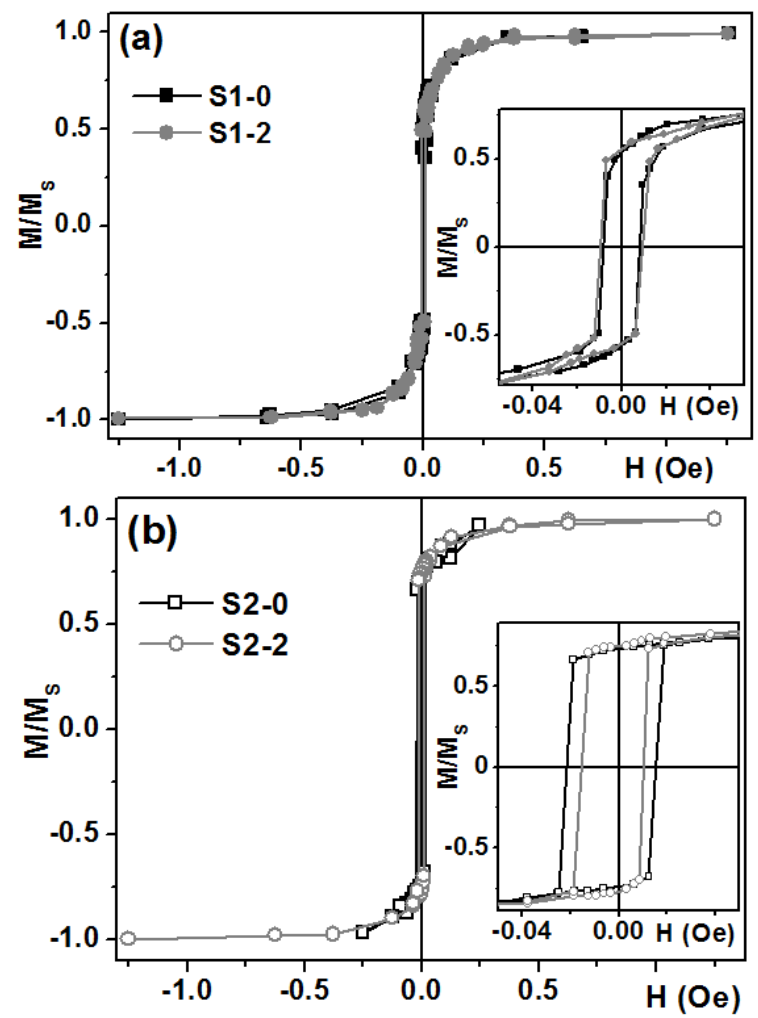

Fig. 2. Longitudinal hysteresis loops of $\mathrm{FeCoCrSiB}$ (a) and $\mathrm{FeCoNiSiB}$ (b) ribbons before and after their treatment in toluene. Insets show curves in a smaller field interval.

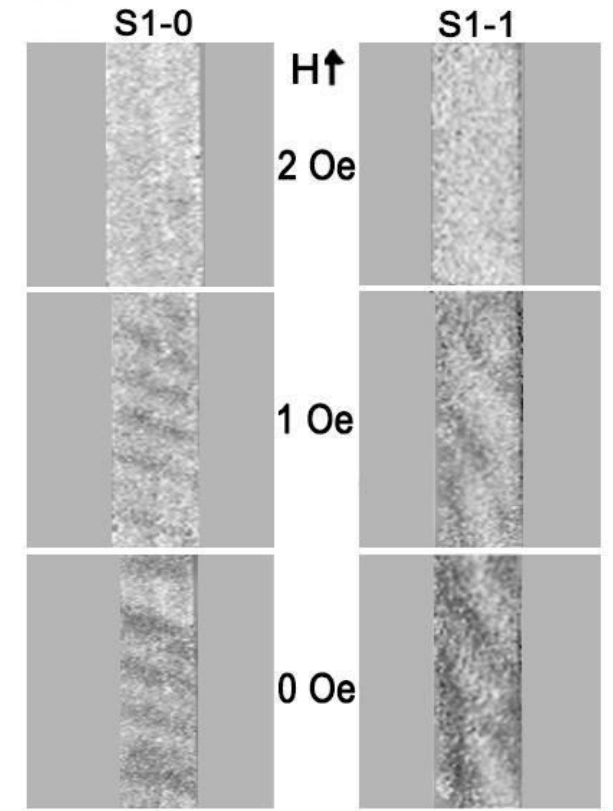

Fig. 3. MOKE images of domain structure of $\mathrm{FeCoCrSiB}$ ribbons before and after surface modification.
Changes of the shape of GMI curve of S1 sample indicate an increase of the contribution of longitudinal component of the effective magnetic anisotropy in a good agreement with MOKE studies. The existence of quenching stresses layers of as-cast quenched ribbons is known [12]. Surface modification in aromatic solvent seems to cause stress relaxation in the near-surface layer due to an appearance of chemical bonds between the carbon covering and a practically oxidized surface of the samples. As the changes of magnetic anisotropy of

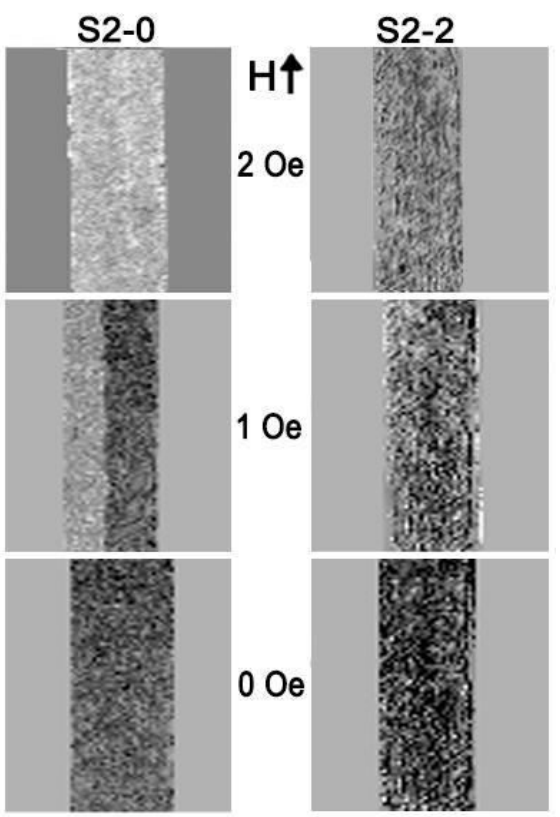

Fig. 4. MOKE images of domain structure of and $\mathrm{FeCoNiSiB}$ ribbons before and after surface modification.
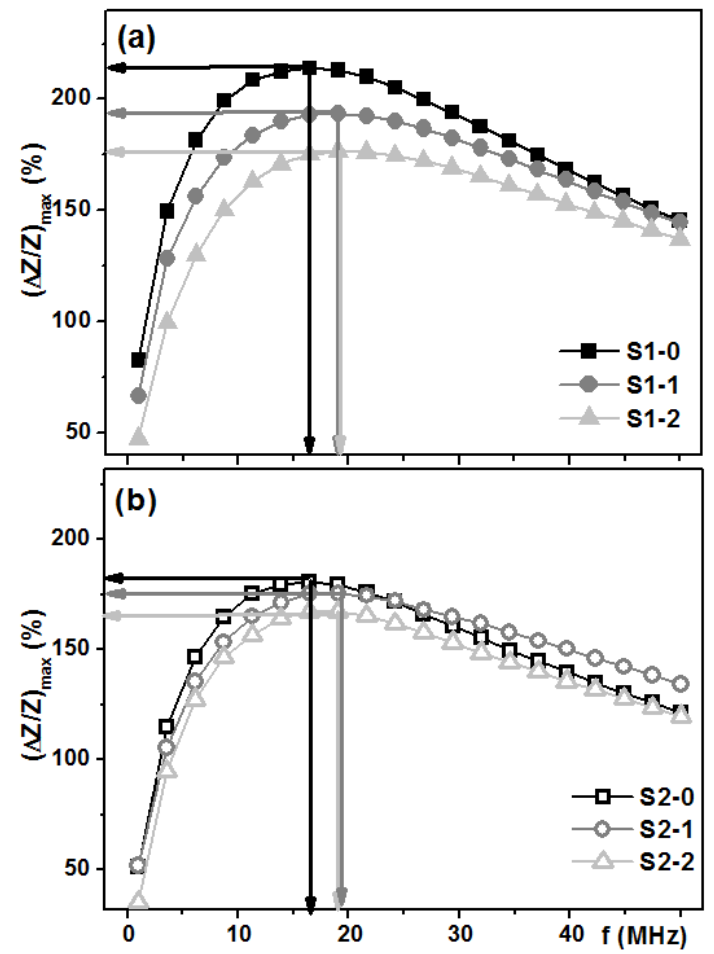

Fig. 5. Frequency dependencies of total impedance ratio for S1 (a) and S2 (b) ribbons before and after treatment in toluene. Arrows indicate positions of the maxima. 
the sample S1 are more significant the decrease of the GMI ratio is much larger than in the $\mathrm{S} 2$ case. Corrosion stability of the samples was studied for $0.9 \% \mathrm{NaCl}$ solution, they were placed into solution and mass loss was systematically measured (Fig. 7). The mass loss is a bit smaller in the case of the ribbon S2-1 with surface passivation by low temperature carbon layer deposition.
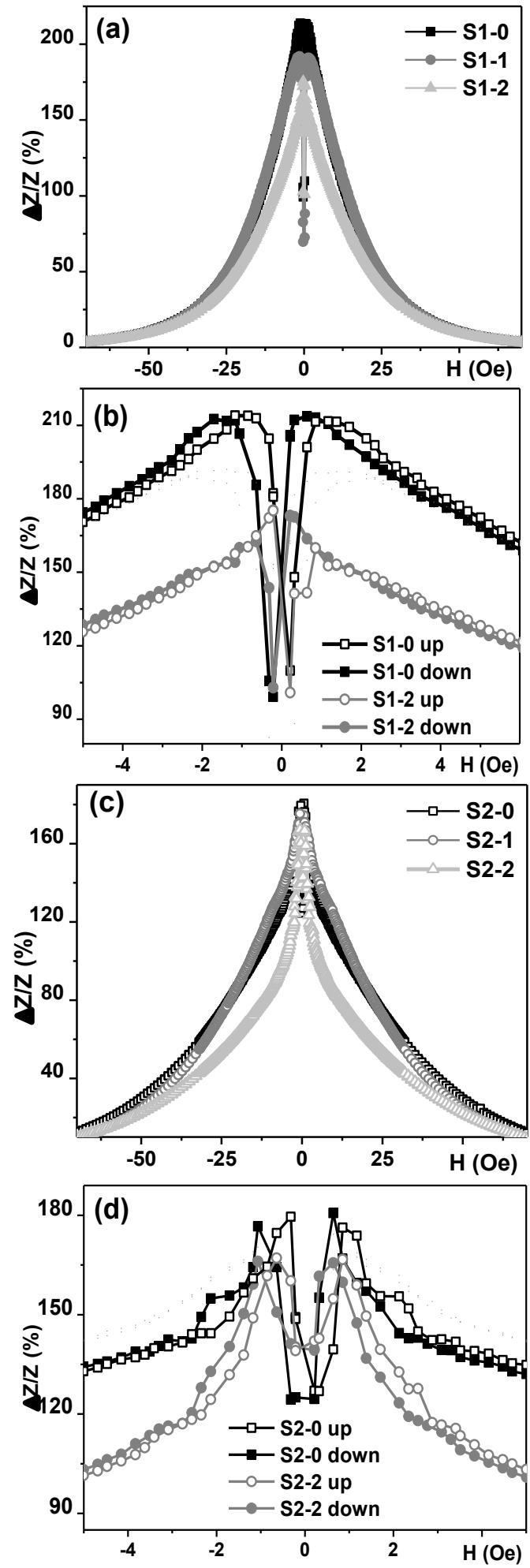

Fig. 6. Field dependences of GMI ratio for $\mathrm{FeCoCrSiB}$ (a) and FeCoNiSiB (b) ribbons before and after surface modification. Decreasing (down) and increasing (up) branches are shown.

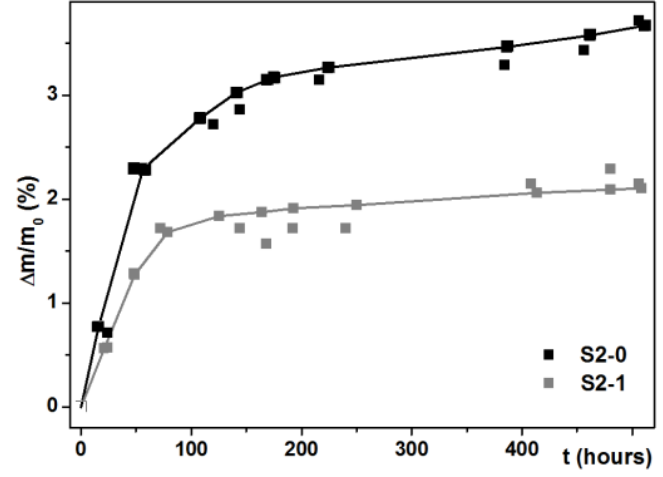

Fig. 7. Relative weight change of $\mathrm{S} 2$ ribbon in $0.9 \% \mathrm{NaCl}$ solution before (S2-0) and after (S2-1) treatment in toluene.

\section{Conclusion}

Room temperature carbon deposition from toluene was confirmed for $\mathrm{Fe}_{3} \mathrm{Co}_{66} \mathrm{Cr}_{3} \mathrm{Si}_{16} \mathrm{~B}_{12}$ and $\mathrm{Fe}_{6} \mathrm{Co}_{60} \mathrm{Ni}_{10} \mathrm{Si}_{14} \mathrm{~B}_{10}$ of amorphous ribbons. Deposition process involves change of the surface magnetic anisotropy in the nearsurface layers and modifies the GMI effect. An increase in corrosion resistance was observed for $\mathrm{FeCoNiSiB}$ ribbon in $0.9 \% \mathrm{NaCl}$ solution. It might be useful as a method for biosensor surface passivation.

\section{Acknowledgments}

We thank UCSU «Modern nanotechnologies» and S.O. Volchkov for special support.

\section{References}

1. A.P.F. Turner, Science 2901315 (2000)

2. D.R. Baselt, G.U. Lee, M. Natesan et al., Biosens. Bioelectron. 13731 (1998)

3. G. Kurlyandskaya and V. Levit, Biosens. Bioelectron. 20 (8) 1611 (2005)

4. J. Devkota, et al, J. Appl. Phys. 113, 104701 (2013)

5. R.S. Beach and A.E. Berkowitz, Appl. Phys. Lett. 643652 (1994)

6. L. V. Panina, K. Mohri, T. Uchiyama, M. Noda, et al. IEEE Trans. Magn. 311249 (1995)

7. A. S. Antonov, S. N. Gadetskii, A. B. Granovskii, et al., Phys. Met. Metal. 83 (6) 612 (1997)

8. X. Liang, X. Li, C. Chen, Z. Liang, at al., Int. J. of Electrochem. Sci. 108395 (2015)

9. A.V. Semirov, M.S. Derevyanko, D.A. Bukreev, at al, J. Magn. Magn. Mater. 41597 (2016)

10. A.P. Safronov, G.V. Kurlyandskaya, A.A. Chlenova et al, Langmuir 303243 (2014)

11. G.V. Kurlyandskaya, D. de Cos, S.O. Volchkov, Russian J. Nondestruct. Test. 45377 (2009)

12. A. Saad, J.A. Garcia, G.V. Kurlyandskaya., J.D. Santos, L. Elbaile, Jap. J. of Appl. Phys. 44 № 7A 4939 (2005) 